\title{
Effects of System Rotation on Turbulence Structure: A Review Relevant to Turbomachinery Flows
}

\author{
JAMES P. JOHNSTON * \\ Thermosciences Division, Department of Mechanical Engineering, Stanford University, Stanford, CA 94305, USA
}

(Received 11 June 1997; Revised 29 August 1997)

\begin{abstract}
Turbomachine rotor flows may be affected by system rotation in various ways. Coriolis and centrifugal forces are responsible for (i) modification of the structure of turbulence in boundary layers and free shear layers, (ii) the generation of secondary flows, and (iii) "buoyancy" currents in cases where density gradients occur. Turbulence modification involves reduction (stabilization) or increase (destabilization) of turbulent Reynolds stresses by Coriolis forces; effects which are of special importance for the understanding and prediction of flows in radial and mixed flow pump and compressor rotors. Stabilization/ destabilization effects are discussed by a selective review of the basic research literature on flows in straight, radial, rotating channels and diffusers.
\end{abstract}

Keywords: Coriolis force, System rotation, Turbulence modification, Channels, Diffusers, Boundary layers

\section{INTRODUCTION}

In 1970 a symposium on "Fluid Mechanics, Acoustics and Design of Turbomachinery" was held a Pennsylvania State University where, in session II, the current state of knowledge on effects of system rotation on boundary layers in turbomachine rotors was reviewed and discussed by the attendees, Johnston (1974). The fact that Coriolis forces cause significant secondary flows (crossflows from pressure to suction side) in the endwall boundary layers of radial and mixed flow pump and compressor impellers had already been clearly established, but direct experimental evidence concerning Coriolis effects on turbulence and transition in boundary layers and free shear layers was new at the time of the symposium. Many of the issues discussed at the Penn State meeting had their origin in questions raised in the early 1960s by R. C. Dean Jr., see Dean (1968) where his observations concerning flow in radial flow compressor impellers are published. It is the objective of this paper to review our knowledge on these subjects, 27 years later. This brief, selective review is not aimed for the research community. Rather, it is intended for the design engineer trying to understand and predict rotor flow phenomena that may at first glance seem unusual.

*Tel.: (415)723-4024. Fax: (415)723-4548. E-mail: Johnston@vonkarman.stanford.edu. 
Rotor flow fields are most conveniently examined in coordinates which rotate with the rotor at steady angular velocity, $\Omega$, about the axis of the machine. The fluid velocity, $\mathbf{U}$, measured with respect to rotating coordinates, obeys the conservation of mass relation,

$$
\frac{\partial \rho}{\partial t}+\nabla \cdot(\rho \mathbf{U})=0
$$

when the fluid density, $\rho$, is variable. However, when $\rho$ is constant, Eq. (1a) reduces to the simple continuity equation,

$$
\nabla \cdot \mathbf{U}=0 .
$$

Note that symbols in bold are vectors, and $\nabla$ is the vector gradient operator which when multiplied (scalar product) into a vector such as $\mathbf{U}$ produces the sum of spatial gradients of the vector. ${ }^{\dagger}$

The dynamic equation of motion for a fluid particle, in steadily rotating coordinates, may be given by

$$
\frac{\mathrm{DU}}{\mathrm{D} t}=2 \mathbf{U} \times \Omega+\nabla \frac{\Omega^{2} r^{2}}{2}+\frac{-\nabla p}{\rho}+\mathbf{F}_{\mathrm{vis}}
$$

$$
\begin{aligned}
& \text { particle }=\text { Coriolis }+ \text { centrifugal }+ \text { pressure } \\
& \text { acceleration force force force } \\
& + \text { viscous } \\
& \text { force }
\end{aligned}
$$

There are four forces (per unit volume) which control the fluid's motion. The Coriolis and centrifugal forces are 'virtual' forces that result from the use of rotating coordinates whereas pressure and viscous forces are the real forces on a fluid particle in any coordinate system. When $\nu \nabla^{2}(\mathbf{U})$ is substituted for $\mathbf{F}_{\mathrm{vis}}$, the incompressible, constant viscosity Navier-Stokes equations are obtained, where $\nu=\mu / \rho$, is the kinematic viscosity.

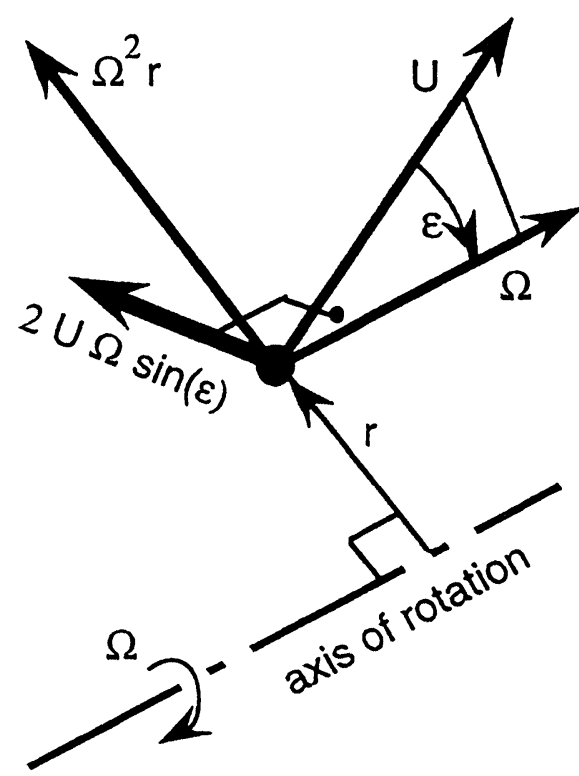

FIGURE 1 Coriolis and centrifugal forces (per unit volume). on a fluid particle.

The centrifugal force has a magnitude of $\left[\Omega^{2} r\right]$ and acts along the local radial direction as shown in Fig. 1. The figure also shows the direction and magnitude, $[2 U \Omega \sin (\varepsilon)]$, of the Coriolis force. This force is perpendicular to a plane formed by the $\mathbf{U}$ and $\Omega$ vectors, and is directed as shown, according to the right hand rule of the vector cross product. The angle $\varepsilon$ ranges from 0 to $\pi / 2$. Since $\varepsilon$ is close to $\pi / 2$ in the case of radial flow rotors, it may be anticipated that Coriolis effects could be large in such cases. For flow in axial flow rotors where the radial component of $\mathbf{U}$ is very small Coriolis forces are also small and they point in the radial direction.

In the case of a constant density flow, the centrifugal force plays no independent role in the dynamics of motion as it may be combined with the pressure force term to form a single term, $-\nabla\left(p^{*} / \rho\right)$ by the definition of an 'effective pressure', $p^{*}=p+\rho\left(\Omega^{2} r^{2}\right) / 2$. In general however, where density gradients, $\nabla \rho$, exist in the flow, the

\footnotetext{
${ }^{\dagger}$ For Cartesian coordinates, $x_{i}(i=1,2$, or 3$)$ where the vector's components are $U_{i}$, the dot (scalar) product gives the sum of deriviatives; $\nabla \cdot \mathbf{U}=\partial U_{i} / \partial x_{i}=\partial U_{1} / \partial x_{1}+\partial U_{2} / \partial x_{2}+\partial U_{3} / \partial x_{3}$. The cross (vector) product, $\nabla \times \mathbf{U}$ represents the curl of the velocity field, and yields the vorticity $(2 \times$ fluid rotation) with respect to rotating coordinates. The vorticity in inertial space (stationary coordinates) is $2 \Omega+\nabla \times \mathbf{U}$. In rotating Cartesian coordinates, the vorticity in the $x_{1}$ direction is $\left(\partial U_{2} / \partial x_{3}-\partial U_{3} / \partial x_{2}\right)$.
} 
centrifugal force is similar to a gravitational body force (neglected here) and thus may contribute to the flow's dynamics.

The Coriolis force has the interesting characteristic that it can't affect the energy of a flow directly. Change of energy of a fluid particle is affected by an applied force, $\mathbf{F}$, according to the work, $\mathbf{F} \cdot \mathrm{dx}$, done on the particle when it is displaced through the distance $d \mathbf{x}$. Since instantaneous displacement in rotating coordinates is along the $\mathbf{U}$ vector, and because $\mathbf{U}$ is perpendicular to the Coriolis force vector there can be no transfer of energy to/from the flow by the Coriolis force. This has the consequence that both the mean flow and the turbulence kinetic energy, ${ }^{\ddagger} k$, cannot be directly produced or destroyed by Coriolis force. However, both the normal stress and the shear stress components of the turbulent Reynolds stress tensor, $-\overline{u_{i} u_{j}}$, may be affected by this force.

\section{Effects of Rotation}

The effects of rotation may be illustrated using a very simple example of a rotor flow, the flow along a radial rectangular channel as depicted in Fig. 2. Typically one expects a core region of high speed flow with lower speed fluid in the end-wall and side-wall boundary layers as shown in the isometric view. An END VIEW, looking along the center line of the channel shows a shaded area representing regions in the boundary layers where the relative velocity, $U$, is low with respect to its value in the core. The Coriolis force is shown in the figure by the heavy arrow marked $2 U \Omega$. Its value will be largest in the core where $U$ is at its maximum value. The core region Coriolis force will establish a pressure gradient between the two side walls causing high pressure, $p_{\mathrm{p}}$, near one (pressure) side wall and lower pressure, $p_{\mathrm{s}}$, near the opposite (suction) side wall. To a degree of approximation
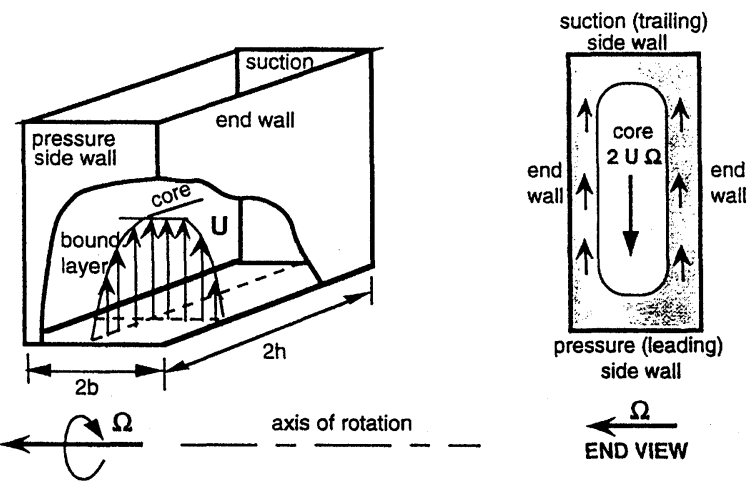

FIGURE 2 Rotating rectangular channel flow with wall boundary layers.

the cross-stream pressure gradient in simple channel flow is

$$
\frac{\left(p_{\mathrm{p}}-p_{\mathrm{s}}\right)}{2 h} \approx \rho(2 U \Omega)
$$

The pressure gradient established in the core will be felt inside the end-wall boundary layers, according to boundary layer theory. Because of the lower flow speed in the boundary layers they will develop lower Coriolis forces, and consequently fluid particles inside the end wall layers must be accelerated and move toward the suction side wall, in the direction indicated by the short arrows near the end walls. This cross-flow is called the secondary flow due to system rotation.

Coriolis driven secondary flows have different relative effects on rotor flows depending on many geometric and flow variables. In the case of simple rectangular channels, various investigations have examined rotational effects, and one finds that channel aspect ratio $(\mathrm{AS}=b / h)$ is an important parameter. Figures 3 and 4 are used to illustrate the point. Two cases, low and high aspect ratio, are sketched in the first figure. They show that secondary streamlines established by the acceleration of the fluid in the end wall boundary layers

\footnotetext{
${ }^{\ddagger}$ Cartesian compact subscript notation is used in discussions of turbulence. $x_{i}(i=1,2$, or 3$)$ represents the $x, y$, or $z$ directions, $U_{i}$ represents $U, V$, or $W$, the time mean or ensemble average components of the velocity, $u_{i}$ represents $u, v$, or $w$, the velocity fluctuations about the mean. $\overline{u_{1} u_{1}}$ is a typical average of the fluctuations along 1 ( $x$-direction), and $k=1 / 2\left(\overline{u_{1} u_{1}}+\overline{u_{2} u_{2}}+\overline{u_{3} u_{3}}\right)$ is the turbulence kinetic energy. $-\overline{u_{i} u_{j}}(i=1,2$, or 3 , and $j=1,2$, or 3$)$ is the Reynolds stress tensor; it has six components, the three shear stresses where $i \neq j$ and the three normal stresses where $i=j$.
} 


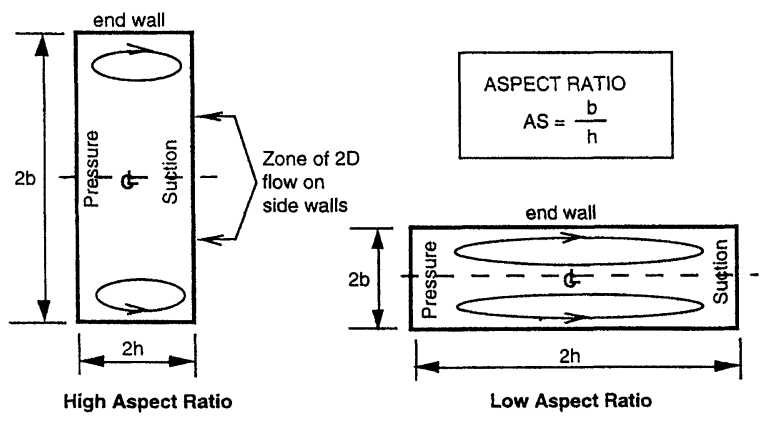

FIGURE 3 Secondary flow streamline patterns in the endwall regions of rotating rectangular channels of high and low aspect ratio.

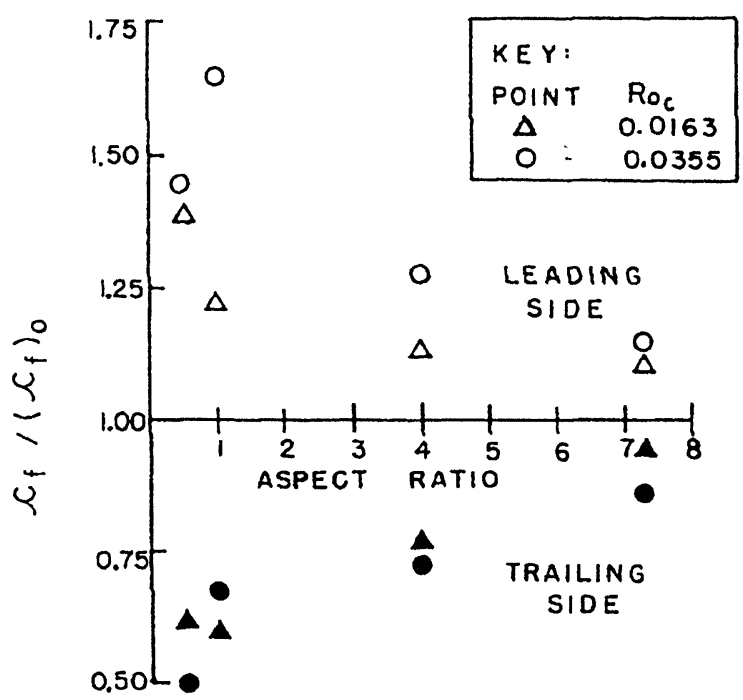

FIGURE 4 Skin friction coefficient, $C_{\mathrm{f}}$, at center line versus channel aspect ratio, $\mathrm{AS}=b / h$, on leading (pressure/destabilized) and trailing (suction/stabilized) side walls. Rotation number, $R o c=2 \Omega \mathrm{h} / U_{\mathrm{m}}$, where $U_{\mathrm{m}}$ is area average flow velocity through the channel. Graph from Moore (1967).

tend to cover the whole cross section of the low aspect ratio channel, but when AS is high, the secondary streamlines are concentrated near the end walls leaving the core region unaffected. Moore (1967) experimented with turbulent boundary layer flow in rotating channels of differing aspect ratio and showed that secondary flows had large effects near the center line of symmetry when AS was low, compared to the effects when AS was high. Figure 4 shows a decrease of the skin friction coefficient, $C_{\mathrm{f}}$, on the leading (pressure) side-wall as AS increases, and vise versa on the trailing (suction) side-wall. Moore also varied the rotation rate to show that the effect increased as rotation rate increased.

A surprising feature of these results is the relatively large effects seen for the channel of largest aspect ratio, $\mathrm{AS} \approx 7.3$. In this case, the secondary flow in regions far from the end walls would be expected to cause $\mathrm{a} \pm 1$ or 2 percent change in side wall skin friction rather than the \pm 10 to 15 percent changes noted by Moore. These large increases or decreases are not secondary flow effects, but they result from destabilization (increase) or stabilization (decrease) of turbulent shear stresses in the side-wall boundary layers themselves. These effects are the main topic of this paper.

In addition to the (i) secondary flow effects and (ii) the turbulence stabilization effects in rotating systems, one needs to be aware that (iii) centrifugal forces can also contribute substantially to the flow. This force is particularly important when large density gradients exist, as might be the case for the flow inside blade cooling passages of gas turbine rotors. The centrifugal force is always radial and acts outward with respect to the axis of rotation, see Fig. 1. Consequently, it causes fluid forces which tend to drive low density fluid toward the axis of rotation and high density fluid away from the axis. Because of the similarity to the effects of gravity in a stationary case, the flows induced by centrifugal forces are sometimes designated as centrifugal buoyancy currents. This is a special topic which will not be discussed further in this paper.

\section{Stability and Instability}

These terms are used here to denote the tendency for a boundary layer or a free shear layer to experience increased or decreased levels of turbulence with respect to the 'natural' levels that would develop under non-rotating conditions. Nonrotating conditions are the basis for models used to estimate the turbulent Reynolds stresses, $-\overline{u_{i} u_{j}}$. These models then provide the equations for closure of the set of mean flow equations, the 
Reynolds average Navier-Stokes (RANS) equations. For example, Johnston and Eide (1976) applied empirical algebraic corrections to a mixing length method in order to model the effects of rotation on the turbulent shear stress, $-\overline{u v}$. Parameters for expressing these effects were derived from knowledge concerning the effects of rotation on flow stability.

A boundary layer velocity profile $U(y)$ on a rotor blade is illustrated in Fig. 5. Here, $2 U \omega$, is the component of the Coriolis force along the $y$-axis which is defined to be perpendicular to the blade surface. $\omega=\Omega \sin (\varepsilon) \cos (\psi)$ is the effective rate of rotation in regard to stabilization effects. For example, in radial flow rotors $\varepsilon \approx \pi / 2$ and $\psi \approx 0$ so $\omega \approx \Omega$. If the direction of the force is positive along the $y$-axis (as drawn), then the boundary layer will be stabilized. Which means that a fluid eddy displaced along $y$ in either the $\pm y$ direction feels a restoring force which tends to return it to its original $y$ location. Conversely, if the effective Coriolis force is in the reverse orientation, pointing toward the wall, displaced eddies feel forces that tend to increase their displacements from their original location, the situation is destabilizing.

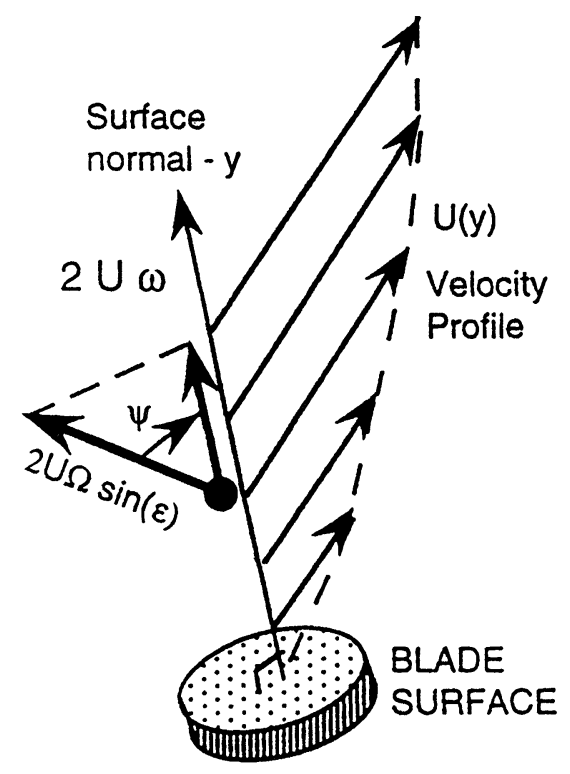

FIGURE 5 Coriolis force on a fluid particle near a suction surface where $\omega$ is effective rate of rotation for stability effects.
Stabilization effects were discussed and proper parameters for their description developed by Bradshaw (1969). His parameter, Ri, has become widely used in all subsequent work. Ri, by analogy named the gradient Richardson number, permits one to describe the degree of stabilization at each $y$ position in a velocity profile where the local velocity gradient is $\partial U / \partial y$.

$$
\mathrm{Ri}=S(S+1), \quad \text { where } S=\frac{-2 \omega}{\partial U / \partial y} .
$$

The flow is locally stabilized when: $\mathrm{Ri}>0$, and it is destabilized when $\mathrm{Ri}<0$. The flow stability conditions are neutral $(\mathrm{Ri}=0)$ for two values of $S$, when $S=0$ and, under rotating conditions, when $S=-1$. This latter condition is interesting in that the vorticity of the velocity profile with respect to inertial (non-rotating) coordinates, $(2 \omega-\partial U / \partial y)$, is also zero for this neutrally stable condition.

In a radial or mixed flow pump or compressor impeller, according to these criteria, the boundary layers on the suction (trailing) sides of the blades will have $\mathrm{Ri}>0$ and hence they will be stabilized, but on the pressure (leading) sides flow will be destabilized by rotation. In the inviscid core region, should one exist, the flow is generally inertially irrotational and thus it is neutrally stable. The consequences of these effects are that increased turbulence and mixing in destabilized (pressure side) boundary layers makes them less likely to separate, but the stabilized layers on the suction side become less turbulent and more prone to flow separation in regions of adverse pressure gradient.

\section{Developments Since 1970}

These are first outlined to give a brief overview on progress in the field over the past quarter century. For the most part, this review will concentrate on results obtained by experiment. However, some recently computed results obtained by direct numerical simulation (DNS) of the Navier-Stokes equations are also discussed. Turbulence modelling 
for use in RANS methods is not reviewed because such models must result from basic understanding which can only come from experiment and/or DNS.

Newtonian viscosity, $\mu$, must be introduced as a fluid property to properly represent viscous forces in either experimental or DNS results. However, because the density, $\rho$, is constant for all the work reviewed here, the only significant fluid property is the kinematic viscosity, $\nu=\mu / \rho$. Thus, for a system of given geometry one may characterize all dimensionless forces, flow rates, etc. in terms of two dimensionless parameters; a Reynolds number, $\operatorname{Re}=V \lambda / \nu$, and a Rotation number, $\operatorname{Ro}=2 \Omega \lambda / V$. $V$ and $\lambda$ are a fluid velocity scale and a length scale respectively. They must be specified according to the needs of the problem at hand. The Rotation number ${ }^{\pi}$ represents a ratio of Coriolis forces to inertia forces (particle accelerations). The magnitude of the Rotation number, $|\mathrm{Ro}|$ increases from zero as rotation increases, and thus is useful to scale the effects of rotation which, to a first approximation, are found to increase (destablization effect) or decrease (stabilization effect) linearly as Ro increases.

Because the effects of rotation are concentrated in sheared regions such as boundary layers, it is useful, in order to understand the physical processes, to take $V=U_{\mathrm{e}}$, the relative velocity at the edge of a boundary layer, and $\lambda=\delta$, the boundary layer thickness. Therefore, $\operatorname{Re}=U_{\mathrm{e}} \delta / \nu$ and $\mathrm{Ro}=2 \omega \delta / U_{\mathrm{e}}$ are physically meaningful definitions of the two controlling parameters. By taking $\partial U_{\mathrm{e}} / \partial y \approx U_{\mathrm{e}} / \delta$ as an estimate of the mean shear in a boundary layer, One obtains an estimate of the mean global stability parameters, $S \approx-$ Ro, and $\mathrm{Ri} \approx \mathrm{Ro}(\mathrm{Ro}-1)$. Johnston (1974) showed that the magnitude of the boundary layer Rotation number is expected to be low $(|\mathrm{Ro}|<0.1)$ in turbomachines even though the effects of rotation are substantial at these low values. Finally, by combining estimates it is seen that the global stability of a rotor blade boundary layer is approximately equal to the Rotation number, i.e., $\mathrm{Ri} \approx-\mathrm{Ro}$. This is a justification for using the Rotation number in turbulence modeling, e.g., see Johnston and Eide (1976).

Although some excellent data sets have been obtained by means of laser anemometry in real compressor and turbine rotors, these flow fields are too complex to allow one to access basic physical phenomena systematically, one at a time. Consequently, basic research investigations have generally employed geometrically simple flows, a number of which are listed in Table I. The cases listed used air or water as the working fluid and were operated at $|\mathrm{Ro}|<0.2$ and at Reynolds numbers which caused boundary layers to be turbulent, conditions characteristic of many turbine and compressor rotor flows. Thirteen experiments and one DNS case utilized radial rotating channels, constant area ducts of rectangular cross section. Three experiments in this group concerned themselves with detailed measurements of mean velocity profiles and Reynolds stresses in the turbulent boundary layers, and two studies addressed the effects of stabilizing rotation on transition to turbulence. Four studies were conducted in twodimensional, plane-walled diffusers. The diffuser flows simulated the effects of adverse pressure gradients along the blades of radial flow pump and compressor impellers where flow separation is often observed on the suction sides of the blades. Some radial, rotating pipe flows and conical diffuser flows are also listed in Table I because they may provide useful information in special applications, such as flow in turbine blade cooling ducts. However, the round cross section of a pipe makes it hard to disentangle stability effects from secondary flow effects. For completeness, two basic experiments on turbulent free shear layers in rotating systems are listed. Finally, the aspect ratios for the various channels and diffuser inlet planes are listed in the right hand column of Table I.

\footnotetext{
I The inverse of the Rotation number, $\mathrm{Ro}^{-1}$, called the Rossby number, is generally used in geophysical fluid dynamics where very high values of Ro are often encountered.
} 
TABLE I Experiments and direct numerical simulations (DNS) of shear flows with system rotation

\begin{tabular}{lc}
\hline & Aspect ratio \\
\hline Developed and developing channel flows & 1 \\
Hill \& Moon ('62) & 2 \\
Moon ('64) & $0.5,1,4,7.3$ \\
${ }^{* *}$ Moore ('67) & 0.37 \\
Wagner \& Velkoff ('72) & 7.3 \\
** Johnston, Halleen \& Lesius ('72) - Fully-developed flow & 0.14 \\
Koyama, Tamura \& Saito ('89) - Fully-developed flow \\
${ }^{* *}$ Kristofferson \& Andersson ('93) - (DNS) - Fully-developed flow & $\infty$ \\
Turbulent boundary layers in straight channels & \\
Koyama, Masuda, Ariga \& Watanabe ('79) & 7 \\
**Watmuff, Writt \& Joubert ('85) & 4 \\
Koyama, Kitagawa, Kangmin ('95) & 0.14 \\
Boundary layer transition in straight channels & 9.8 \\
Matsubara \& Masuda ('89, '91) & \\
Plane walled (2D) diffusers, separation & 0.85 \\
Fowler ('68) & 1 \\
Moore ('73) & 7.3 \\
${ }^{* *}$ Rothe \& Johnston ('76) - Fully-developed flow inlet flow & 4 \\
Ibal \& Joubert ('90,'92) & \\
Back step diffusers, shear layer reattachment \\
${ }^{* *}$ Rothe \& Johnston ('79) \\
Curved walled (2D) diffusers \\
Kikuyama, Murakami, Oda \& Gomi ('83, '89) \\
Pipes and conical diffusers \\
Ito \& Nambu ('71) - Fully-developed pipe flow \\
Murakami \& Kikuyama ('76) - diffuser \\
Kikuyama, Murakami \& Matsumoto ('80) - diffuser \\
Free shear layer flows & $15,5,2$ \\
Bidokhti \& Tritton ('92) - a 2D, two-stream mixing layer & \\
Witt \& Joubert ('85) - a wake from a rod along the axis of rotation & 2 \\
\hline & \\
\hline
\end{tabular}

\section{FOUR SITUATIONS}

The effects of rotation on stabilization are discussed by reference to four distinct situations using five cases from Table I $\left({ }^{* *}\right)$, all chosen because stability effects dominate and the effects of secondary flows are negligible. These cases have high values of channel aspect ratio, AS $\geq 4$. The situations are: (i) fully-developed flow in long rotating channels; (ii) turbulent boundary layers on the walls of a straight rotating channel; (iii) plane walled two-dimensional diffusers with rotation axis perpendicular to parallel end-walls, and (iv) back-step diffuser flow with separation at the step's sharp edge and reattachment downstream. Figure 6 illustrates the general configurations of these four situations.

\section{(i) Fully-Developed Flow in Long Rotating Channels}

This is an extensively studied case, for which the experiments of Johnston et al. (1972), and a direct numerical simulation (DNS) by Khristofferson and Andersson (1993) comprise the useful data base on stabilization effects. For these fully developed flows the boundary layer thickness $\delta \approx h$, half the width of the channel. Also, the appropriate scaling 


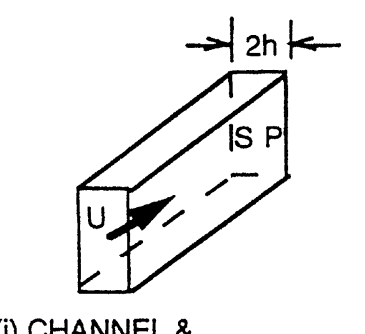

(i) CHANNEL\&

(ii) BOUNDARY LAYER

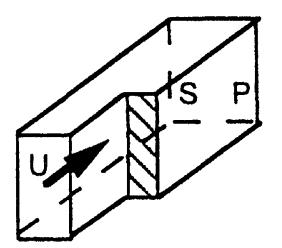

(iv) STEP DIFFUSER

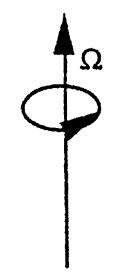

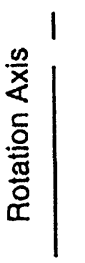

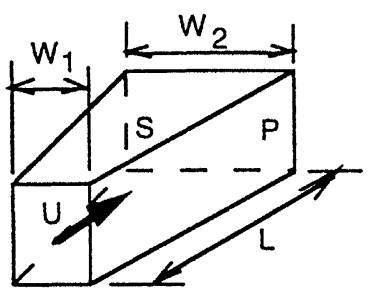

(iii) PLANE DIFFUSER

$A R=\frac{W_{2}}{W_{1}}=1+\frac{L}{W_{1}}(2 \tan \theta)$

Key:

"S" = Suction (Trailing) Side

$" P^{\prime \prime}=$ Pressure (Leading) Side

FIGURE 6 Diagrams illustrating four special situations. Note: S and P side-walls are inverted if $\Omega$ vector reverses direction.

velocity is the mean velocity in the channel, $U_{\mathrm{m}}$. Therefore, the physically significant parameters are defined as: $\operatorname{Re}=h U_{\mathrm{m}} / \nu$ and $\mathrm{Ro}=2 \Omega h / U_{\mathrm{m}}$. The DNS was conducted at $\mathrm{Re}=2900$ and $0 \leq$ $|R o| \leq 0.5$. The experimental results covered a wider range of Reynolds numbers but narrower ranges of Rotation numbers:

$$
\begin{aligned}
& \text { from } \operatorname{Re} \approx 5500 \text { with } 0 \leq|\mathrm{Ro}| \leq 0.2, \\
& \text { up to } \operatorname{Re} \approx 18,000 \text { with } 0 \leq|\mathrm{Ro}| \leq 0.1 .
\end{aligned}
$$

The flow visualization results from Johnston et al. (1972) and Lezius and Johnston (1976) clearly showed how rotation changes the details of the turbulent flow on suction and pressure surfaces. Results from the 1993 DNS study confirm almost all the experimental observations from the early 1970s. In summary, it is found that:

(a) On the stabilized (suction) side increase of $\mid$ Ro $\mid$ decreased turbulence activity continuously, at all Reynolds numbers. For $\operatorname{Re} \approx$ 5500 , when $|\mathrm{Ro}| \approx 0.2$ the stabilized wall layer was so stable that the large energetic structures, called roll cells (described below), which crossed over from the pressure side of the channel could excite only a rapidly damped transition response near the suction wall. At higher Re values, the effect was less dramatic, the flow in the stable side wall layers remained turbulent, but with reduced turbulent shear stress, $-\overline{u v}$, and a drop in turbulence mixing as indicated by lowering of $v$, the component of turbulence normal to the wall, along $y$.

(b) On the unstable (pressure) side the opposite effect is seen, turbulent shear stress and mixing increased steadily as $|\mathrm{Ro}|$ increased.

(c) In the unstable regions of the flow a particular instability phenomenon was observed, the development of large scale roll cells. Such cells are predicted by application of hydrodynamic stability theory for low Reynolds number, laminar, rotating channel flow, and the cells have some of the characteristics of TaylorGörtler (T-G) vortices, ${ }^{\S}$ see Hart (1971) and Lezius and Johnston (1976). However, in turbulent channel flows the cells appeared as a quasi periodic pattern of eruptions which moved away from and perpendicular to the pressure wall. The spanwise spacing of these eruptions was roughly equal to the channel half width, $h$. Between the outward, rather

${ }^{\S} \mathbf{T}-\mathrm{G}$ vortex cells have weak secondary ( $y-z$ plane) circulations about axes along the mainflow direction. 
turbulent eruptions, calmer fluid from the suction side of the channel returned slowly to the unstable region. An individual roll cell was defined as the combination of half an eruption and half a calm return flow. The cells were never stationary in space, but tended to continuously breakup and reform in different locations over time. Compared to the short lived, smaller scale and very chaotic turbulence, the more organized roll cells had comparatively long time scales, and hence were thought of as quasi stationary events.

The consequence of these changes in flow structure are seen by examination of a few quantitative measurements. Mean velocity profiles from both the experiments (a) and from the DNS results (b) are shown in Fig. 7. It is clear that changes in turbulence structure are having major effects on the mean velocity distributions, even for very small values of $|\mathrm{Ro}|$. The straight lines drawn tangent to the central portions of each profile have slopes that correspond to the condition of irrotational flow with respect to stationary coordinates. The zones where the data points lie on these lines are zones of neutral stability, according to the stability criteria set out above. As $\mid$ Ro $\mid$ increases, the neutrally stabile portion of a profile increase in length, a result that has been seen in other fully-developed turbulent flows where roll cell or Taylor-Görtler vortices can develop. ${ }^{* *}$ Flow visualization suggests that the roll cells are the primary cause of the region of neutral stability; it is observed that the roll cell eruptions increased in strength as $|\mathrm{Ro}|$ increased.

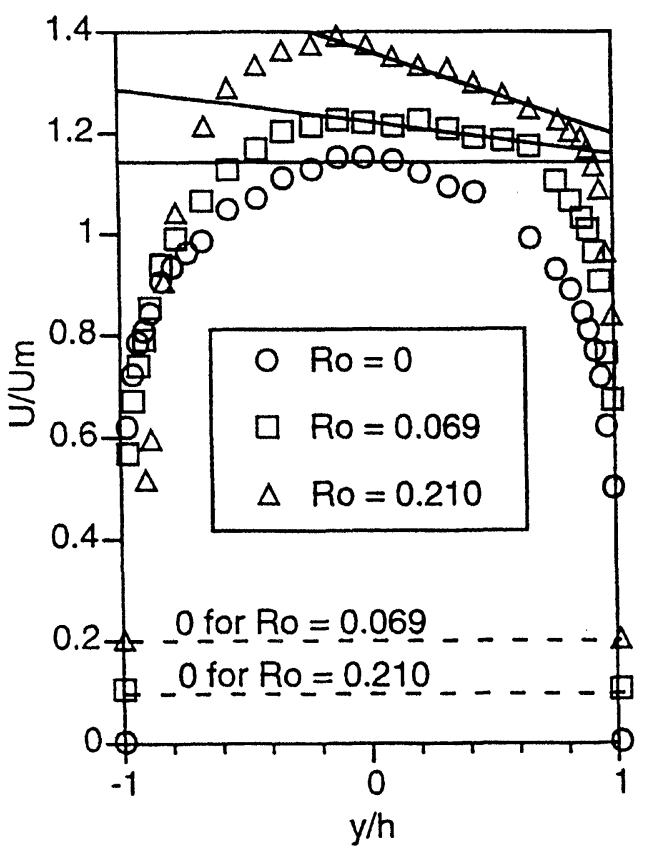

(a) $\operatorname{Re}=5,500$

Johnston et al. (1972)

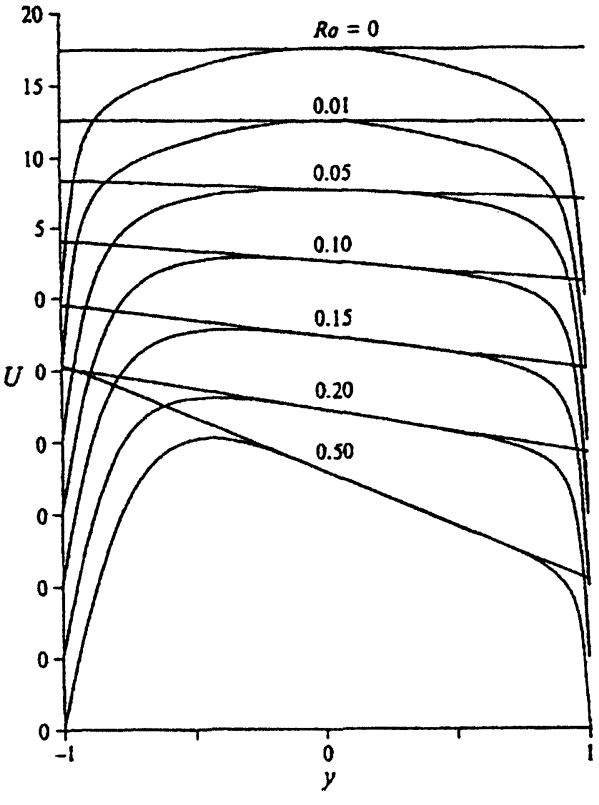

(b) $R e=2,900$

Kristofferson \& Andersson (1993)

FIGURE 7 Mean velocity profiles for fully-developed turbulent channel flows. Notes: The stabilized side is at $y / h=-1$ and destabilized side at $y / h=+1$. In (b), U nondimensionalized using $u_{\tau}$, the global wall shear velocity (see reference).

\footnotetext{
${ }^{* *}$ Flow in a long channel of constant width with constant inner and outer wall curvatures, and flow in the gap between concentric cylinders where the inner cylinder rotates are primary examples.
} 


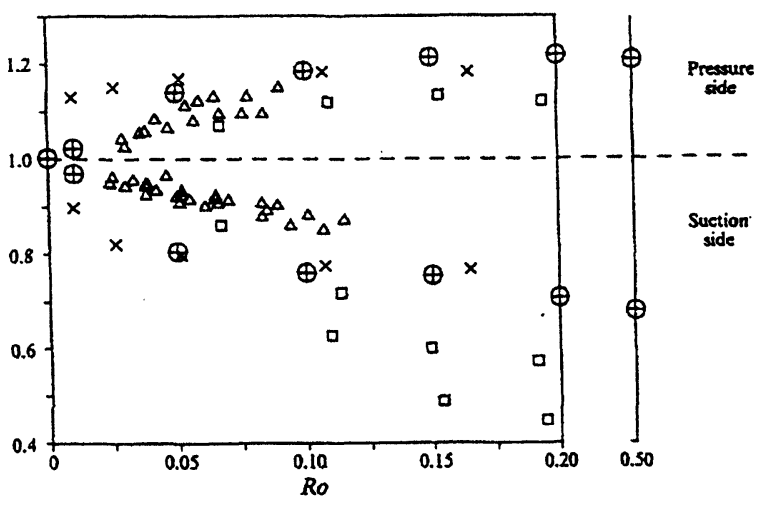

FIGURE 8 Mean wall friction velocities, $u_{\tau \mathrm{p}} / u_{\tau}$ (pressure side), and $u_{\tau \mathrm{s}} / u_{\tau}$ (suction side), versus $|\mathrm{Ro}|$. Symbols: $\oplus$, DNS, $\operatorname{Re}=2900 ; \square$, Johnston et al. (1972), $\operatorname{Re} \approx 5500 ; \Delta$, Johnston et al., $11700<\operatorname{Re}<18000 ; \times$, LES by Miyake \& Kajishima (1986). From Kristofferson \& Andersson (1993).

Figure 8 illustrates the effects of rotation on the wall skin friction stresses in terms of the wall friction velocities, $u_{\tau \alpha}=\left(\tau_{\text {wall } \alpha} / \rho\right)^{0.5}$, on suction ( $\alpha=\mathrm{s})$ or pressure $(\alpha=\mathrm{p})$ walls. The global average value, $u_{\tau}$, used for normalization is based on the streamwise pressure gradient, $-\mathrm{d} p^{*} / \mathrm{d} x$. Wall friction rises continuously on the pressure wall, but appears to level out at higher $\mid$ Ro $\mid$ values. On the suction wall, at higher $|\mathrm{Ro}|$ wall friction drops to quite low levels. There is evidence in the flow visualization that damped laminar flow may exist in a thin layer near the suction surface at the highest $|\mathrm{Ro}|$ values for the lowest Reynolds numbers. The results from a recent large eddy simulation (LES) are also shown on Fig. 8, and except for values of $|\mathrm{Ro}|<0.05$, these results seem to be in good agreement with the rest.

Other computed results, using various turbulence models (not reviewed here) have also been compared to the data shown in Figs. 7 and 8 and fairly good agreement has been attained when the levels of Ro are low. Kristofferson and Andersson (1993) and a more detailed analysis of the CFD results by Andersson and Kristofferson (1993), provide the best current information for successful turbulence modeling of turbulent boundary layer and channel flows with moderate to high system rotation strengths, Ro up to \pm 0.5 .

\section{(ii) Turbulent Boundary Layers on the Walls of Straight Rotating Channels}

These were studied by two groups, Koyama et al. (1979, 1995) and Watmuff et al. (1985). The discussion concentrates on Watmuff's case as his experiment simulates conditions of a two-dimensional turbulent boundary layer with zero free stream pressure gradient. The pressure gradient was controlled by adjustment of the angle between channel side walls so that the free-stream velocity was constant from inlet to exit along the whole length of the side wall on which boundary layers were measured. Secondary flow effects from the end-wall cross flows were negligible in the central regions of the test surface because the boundary layers were thin. They grew, downstream of a trip, to a maximum thickness $\delta \approx 25 \%$ of the channel width, $2 h$, and thus the effective aspect ratio was twice the actual ratio defined by the walls, $\mathrm{AS}=4$.

Watmuff's air flow study reports hot wire (turbulence) and total head tube (mean velocity) profiles at five downstream stations for six different flow conditions. He used three free stream velocities $\left(U_{\mathrm{e}}=7.5,10\right.$, and $\left.15 \mathrm{~m} / \mathrm{s}\right)$ and three rotation rates (stabilizing at $\Omega=-60 \mathrm{rpm}, 0 \mathrm{rpm}$, and destabilizing at $+60 \mathrm{rpm})$. The Reynolds numbers, where $\operatorname{Re}=\delta U_{\mathrm{e}} / \nu$, and magnitudes of the Rotation numbers, where $|\mathrm{Ro}|=2|\Omega| \delta / U_{\mathrm{e}}$, achieved maximum values at the downstream station where $\delta \approx 35 \mathrm{~mm}: \quad \operatorname{Re}_{\max } \approx 1.7 \times 10^{4}$ to $3.5 \times 10^{4}$ and $|\mathrm{Ro}|_{\max } \approx 0.06-0.03$ over the range of free stream speeds and rotation rates. These parametric values are approximately the same as those encountered in the boundary layers over the blades of radial flow compressors, as noted in Johnston (1974).

Many of the general conclusions drawn from the fully-developed rotating channel cases were seen in this case too, i.e., stabilizing rotation reduced turbulence and wall shear stress, and destabilizing rotation had the opposite effect. Also, the large scale roll cells were observed in the destabilized boundary layer. However, in Watmuff's cases the roll cells tended to be much steadier in space and time than seen previously in the rotating channel 
flows. Another important difference from earlier channel flow studies is that the roll cells did not interact with the boundary layer on the stabilized side, because a thick, inviscid core region separated the suction and pressure side boundary layers by at least $2 \delta$ in all cases.

Some of the most important practical contributions from the study concern the use of scaling parameters to describe the mean velocity profiles. For example, in practical computations one is often limited in the density of mesh points that may be used inside thin boundary layer zones. Therefore, it is necessary to prescribe empirical wall functions in order to satisfy boundary conditions at a point close to a solid wall rather than apply no-slip conditions at the wall itself, $y=0$. Typically, this inner boundary point is at $y / \delta \approx 0.05-0.1$, inside a zone of overlap between inner and outer layers. The overlap region often called the "universal log law" region, because, by use of wall layer scaling, ${ }^{\dagger \dagger}$ the mean velocity profile in the overlap layer generally tends to follow the formula:

$$
U^{+}=\frac{1}{\kappa} \ln \left(y^{+}\right)+C+\Delta U^{+}
$$

For non-rotating, turbulent boundary layers, the constants are usually given as $\kappa=0.41, C=5.0$, and the offset, $\Delta U^{+}$, is set to zero for smooth surfaces and low turbulence free stream flows.

Some mean velocity profiles are shown in Fig. 9 to illustrate the general effects of rotation on the profile shapes. The upward shift in the outer regions for the stabilized case is caused by reduction of turbulent shear stress and vice versa for the destabilized case. The log region is very short for the stabilized case and quite a bit longer in the destabilized flow. Reduction in size of the logarithmic overlap zone is generally in indication of a trend toward lower turbulence and reduced mixing.

The offset parameter, $\Delta U^{+}$, depends on many effects such as degree of surface roughness, free stream turbulence level, etc. For Coriolis force

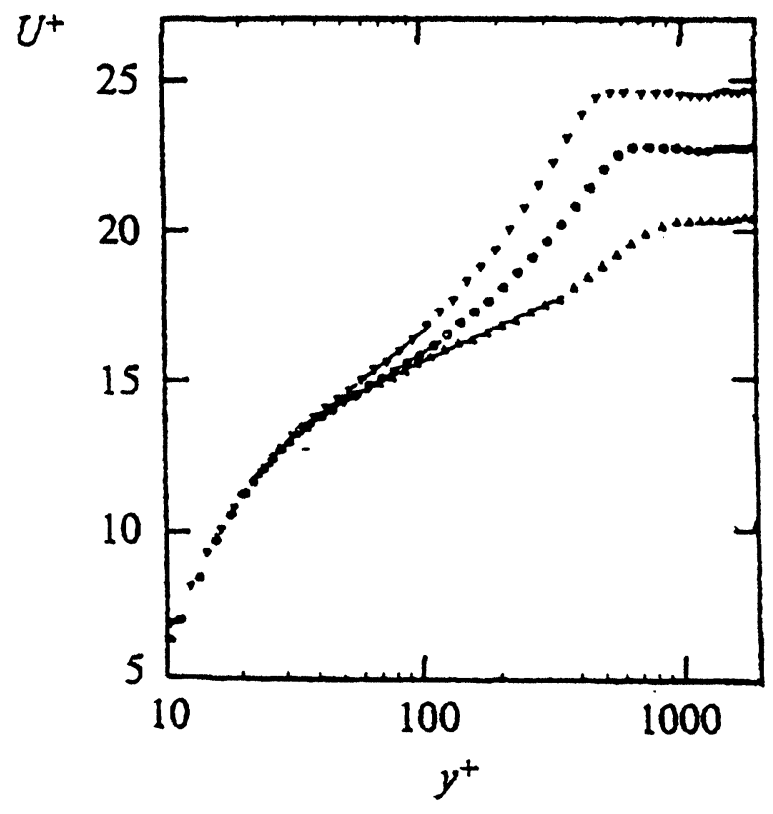

FIGURE 9 Turbulent bondary layer mean velocity profiles from Watmuff et al. (1985). $U^{+}=U / u_{\tau}$ and $y^{+}=y u_{\tau} / \nu$. $U_{\mathrm{e}}=7.5 \mathrm{~m} / \mathrm{s}$, at station 5. UPPER, stable $(-60 \mathrm{rpm})$. MIDDLE, $(0 \mathrm{rpm})$. LOWER, unstable $(+60 \mathrm{rpm})$.

effects, Bradshaw (1969) and others have suggested, on the basis of stability arguments, that one may prescribe the offset using the simple linear relation

$$
\Delta U^{+}=-\beta \frac{2 \omega y}{u_{\tau}}
$$

where $\omega$ the effective rotation rate of Fig. 5, is used here. $\omega$ is negative for a suction (stable) side boundary layer and $\omega$ is positive for a pressure (unstable) side layer. An empirical "constant" $\beta=4 \pm 2$ fits the overlap zone in the channel flow data of Johnston et al. and Watmuff's unstable side profiles fairly well. However, Watmuff's stable side velocity profiles fit the linear offset relation very poorly, if at all. This observation, together with the wide range of possible values of $\beta$ makes the $\Delta U^{+}$ method highly uncertain and therefore not very useful as a Coriolis force correction to the log law.

Both Koyama et al. (1979) and Watmuff et al. suggest a better empirical fit in the overlap zone

\footnotetext{
${ }^{\dagger \dagger}$ Wall layer scaling uses $u_{\tau}$ for velocity and $\nu / u_{\tau}$ for length, so dimensionless velocity is $U^{+}=U / u_{\tau}$ and dimensionless distance from the wall is $y^{+}=y u_{\tau} / \nu$.
} 


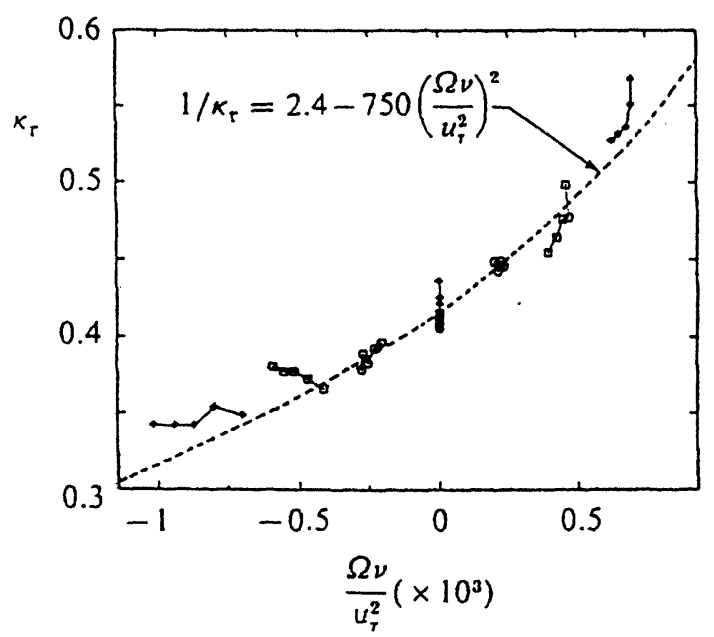

FIGURE 10 Variation of modified log law constant, $\kappa$, from Watmuff et al. (1985) over wide range of conditions.

where $\Delta U^{+}=0$ but $\kappa$ and $C$ are no longer held constant. Both suggest that $\kappa$ and $C$ should be expressed as functions of a Rotation number based on wall layer scaling, $\omega \nu / u_{\tau}^{2}$. For example, Watmuff et al. produced a formula,

$$
\frac{1}{\kappa}=2.4+750\left(\frac{\omega \nu}{u_{\tau}^{2}}\right)
$$

that fits the data quite well, as is seen in Fig. 10. A similar result would be obtained for the variation of $C$ with $\omega \nu / u_{\tau}^{2}$. From a practical vantage, this method is probably the best idea to date for a wall function in rotating boundary layers.

\section{(iii) Plane Walled Two-Dimensional Diffuser Flows with Rotation Axis Perpendicular to the Parallel End-Walls}

These approximate the flows seen in the bladed rotors of radial flow pumps and compressors. The simplest cases are the four experiments on straightwalled diffusers noted in Table I. Unfortunately, Fowler (1968) did not document boundary layer regions in detail, and, like Moore's (1973) rotating diffuser flow, Fowler's experiment was conducted with a low aspect ratio at the diffuser's inlet. Because of the low aspect ratios, these two cases show substantial end-wall secondary flow effects which tend to confuse the interpretation of the data in regard to stabilization effects. Despite this, Eide and Johnston (1976) were able to provide reasonable predictions for the streamwise distribution of boundary layer integral thickness parameters in the upstream, unseparated sections of Moore's diffuser flows. Fortunately, there are two sets of experiments, Rothe and Johnston (1976) and Ibal and Joubert (1993), on straight diffusers at high enough inlet aspect ratios so that end-wall secondary flow effects are of minor importance.

Rothe and Johnston $(1975,1976)$, used the fullydeveloped channel flow of Johnston et al. as a diffuser inlet condition. The diffuser side-wall length, $L$, and inlet throat width, $W_{1}$, were fixed, but the side-walls were hinged at the inlet plane so that $W_{2}$, the outlet width could be changed. Consequently, as the side-wall opening angle, $2 \theta$, was changed the diffuser area ratio, $\mathrm{AR}=W_{2} / W_{1}$, could be varied. Studies were carried out with water flow which facilitated visualization using dye injection methods. Observation of the motion of the injected dye tracers permitted evaluation of the onset of the various stalled flow regimes (unseparated or no appreciable stall, corner stall, 2D stall, and full stall) as the magnitude of the as area ratio and rotation were increased in small steps. The results are shown in Fig. 11 where it is seen that the various regimes of stall set in at increasingly smaller area ratios as rotation rate increases. Boundary layer separation at the upstream end of a stalled region, always occurred on the suction wall, the wall with the stabilized, lower turbulence boundary layer. Stall is first seen in the corners formed at the intersections of end-walls and the suction (stabilized) side-wall. When $\mathrm{Ro}_{Q}$ (defined below) increases at fixed AR (or $2 \theta$ ) pockets of "corner stall" grow and then spread obliquely until the whole suction side-wall separates near the exit plane to form a "two-dimensional stall" zone. As $\mathrm{Ro}_{Q}$ continues to increase, the 2D separation line at the front of this zone moves upstream until it is very near the inlet plane. At this condition, we say the diffuser in a state of "full stall". 


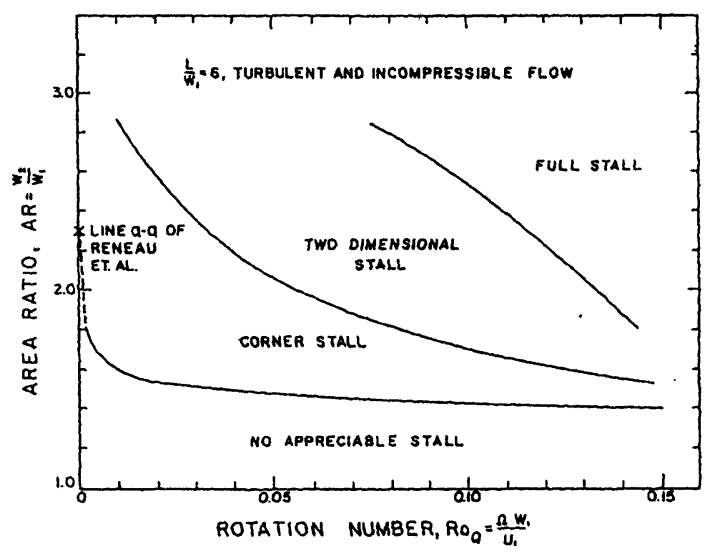

FIGURE 11 Separation flow regimes in the rotating diffusers of Rothe and Johnston (1976).

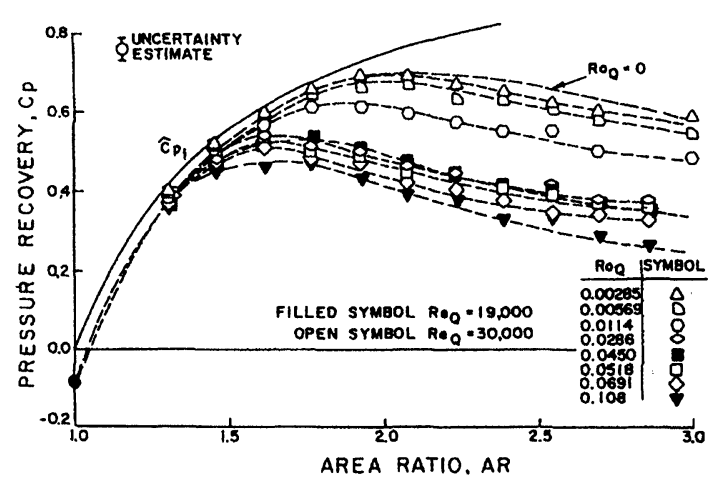

FIGURE 12 Static pressure recovery coefficient, $C_{\mathrm{p}}$, in rotating diffusers of Rothe and Johnston (1976).

In addition to the flow visualization to determine the stalled states, the static pressure distributions along the side-walls were also measured. From these data, the overall (outlet to inlet) pressure rise (recovery) was obtained. It is plotted versus AR in Fig. 12 in terms of the pressure rise coefficient from inlet to outlet:

$$
C_{p}=\frac{p_{2}^{*}-p_{1}^{*}}{\frac{1}{2} \rho U_{1}^{2}}
$$

Here, the inlet mean velocity was based on volume flow rate, $Q$, and inlet cross section area, $A_{1}$;
$U_{1}=Q / A_{1}$. Also, the system Reynolds number and Rotation numbers were defined by $\operatorname{Re}_{Q}=U_{1} W_{1} / \nu$ and $\operatorname{Ro}_{Q}=\Omega W_{1} / U_{1}$ respectively. Pressure recovery attains a maximum (peak) value at a given area ratio for fixed Reynolds number and Rotation number, and the magnitude of $C_{p}$ is reduced as Rotation number rises. As $\mathrm{Ro}_{Q}$ increases the peak is located at successively lower AR values; a trend that correlates with the results of the stall flow regime evaluation given in Fig. 11.

Ibal and Joubert (1993) investigated low speed $(10 \mathrm{~m} / \mathrm{s})$ air flows in diffusers that had thin, turbulent boundary layers at the inlet plane. They used the rotating boundary layer channel and general flow conditions of Watmuff et al. (see Section ii, above) to set up two cases: (i) a very lightly loaded, unstalled diffuser flow with opening angle, $2 \theta=3^{\circ}$, and (ii) a wider angle diffuser with $2 \theta=8^{\circ}$. The wide angle diffuser had substantial 2D boundary separation on the suction (stabilized) surface when it rotated at $40 \mathrm{rpm}$, but it showed no separation when there was no rotation. This research study emphasized the detailed measurement of the growth of the side-wall boundary layers at several rotation rates in the adverse pressure gradients provided by the two diffuser configurations. In general, the observations and conclusions of Rothe and Johnston, and Watmuff et al. also apply here.

Observation \#3 from Ibal and Joubert is worth quoting: "The separated region after two-dimensional separation was steady and relatively quiet." The same remark also applies to the results of Rothe and Johnston. It is of particular relevance for radial compressor rotor flows where steady separated regions called 'wakes' have been observed by various investigators, even in rotor passages of low aspect ratio. The reason for this behavior is that the free shear layer which separates the slowly recirculating fluid in the stalled region from the higher speed through flow ${ }^{\ddagger \ddagger}$ has the same sign of $\partial U / \partial y$ as the boundary layer on the suction

\footnotetext{
${ }^{\sharp \ddagger}$ In the literature on radial flow compressor rotor flows the separated region is call the 'wake' and the higher speed flow that passes over the wake region is called the 'jet', after the designations originated by Dr. R.C. Dean, Jr.
} 
side, and thus it is stabilized by rotation, and at the operating Reynolds numbers it may never undergo free shear layer transition. This phenomenon will be discussed further below.

\section{(iv) Back-Step Diffuser Flows with Separation Forced at the Step's Sharp Edge and Reattachment Downstream}

Back-step diffuser flows with separation forced at the sharp edge of the step, and reattachment on the downstream wall, behind the separated region were studied experimentally by Rothe and Johnston (1979). The diffusers rotated about an axis perpendicular to their parallel end-walls, see (iv) in Fig. 6. In these cases, all the diffusers had the same area ratio $\left(W_{2} / W_{1}=2\right)$, but each had a different aspect ratio (AS $=b / W_{1}=2,5$, and 15). The experiments where carried out in our water flow channel at various Reynolds numbers from $\operatorname{Re}_{Q}=3000$ 28,000 , and for a range of Rotation number magnitudes; $\left|\mathrm{Ro}_{Q}\right|=0-0.15$ (destabilizing), and $\left|\mathrm{Ro}_{Q}\right|=0-0.09$ (stabilizing).

Stabilization is defined here, as above, in terms of the sign of the velocity gradient across the free shear layer that divides the faster through flow from the slowly recirculating fluid in the separated region behind the step. When the free shear layer is destabilized, rotation causes the step side-wall to be a pressure (leading) side, and the boundary layers all along the step side-wall are also destabilized. The case is reversed when rotation is reversed to stabilize the free shear layer. In a faired wall diffuser, and in a radial flow rotor flow, only the stabilized situation exists as separation always starts in the suction (stable) side-wall boundary layer.

Visualization was conducted by injection of dye at the solid walls and by generation of streak lines using hydrogen bubbles generated from fine platinum wires. Analysis of hundreds of individual frames of movie film enabled us to determine the mean (average over a long time) location of reattachment, $X_{\mathrm{R}}$, at the downstream edge of a separation region. Fluctuations of $X_{\mathrm{R}}$, about the mean, were noticed even in stabilized cases because

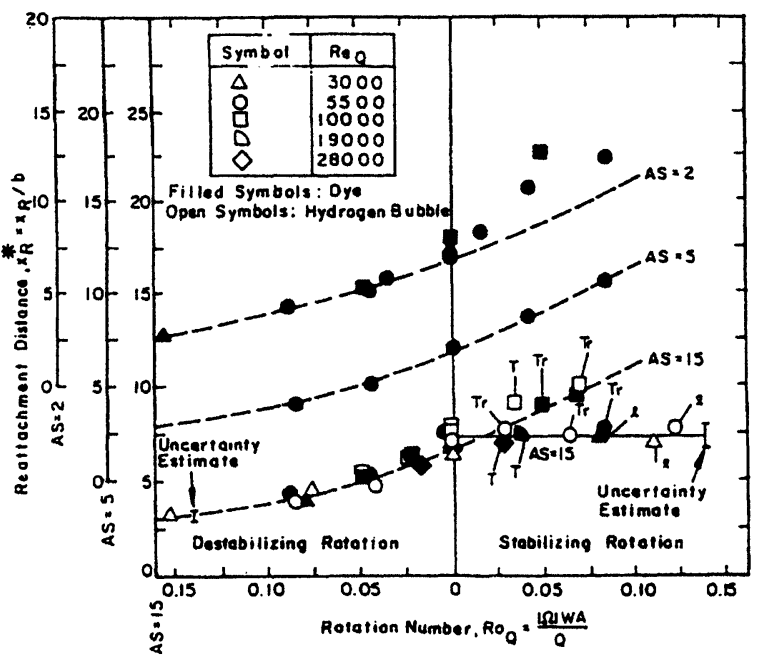

FIGURE 13 Reattachment distance downstream of the step in step diffusers of Rothe and Johnston (1979) versus Rotation number. Reattachment distance, $X_{\mathrm{R}}$, is normalized on step height, $b$.

Coriolis induced stabilization does not act to change the 2D, Kelvin-Helmholtz waves, it only damps three-dimensional, small-scale breakdown, the observed result of transition to turbulence in free shear layers.

The measured values of $X_{\mathrm{R}}$ are plotted versus Rotation number in Fig. 13. With destabilizing rotation, turbulence in the free shear layer increases and entrainment of fluid from the recirculating separated region into the through flow is enhanced. As a result, the flow reattaches closer to the step, i.e., $X_{\mathrm{R}}$ decreases as $\left|\mathrm{Ro}_{Q}\right|$ increases; a result that appears to be independent of diffuser aspect ratio (left hand side of Fig. 13).

On the other hand, the situation is considerably more complex for stabilized free-shear layers as a somewhat different pattern of events is seen for each aspect ratio (right hand side of Fig. 13). In this case, secondary flows near the end-walls are driven into the separated region, an effect opposite from the turbulent entrainment of fluid out of this region. Thus, secondary flows tend to increase, $X_{\mathrm{R}}$, as more fluid is introduced into the separated region causing its volume to increase. This effect must be particularly strong at low aspect ratio where end-wall flows are more important, and it 
seems to justify the results in Fig. 13 where $X_{\mathrm{R}}$ grows rather long for the case of $\mathrm{AS}=2$.

With stabilization, only the $\mathrm{AS}=15$ diffuser is essentially free of end-wall secondary flow effects. In this diffuser, the mixing layer remained laminar or was transitional at the lower Reynolds numbers and entrainment in these cases was probably controlled by growth of the vorticies that are the result of the 2D Kelvin-Helmholtz instabilities. Consequently, at low Reynolds numbers, reattachment location did not change much from its location with zero rotation. One set of data, at $\operatorname{Re}_{Q} \approx 10,000$ clearly shows an increase of $X_{\mathrm{R}}$ as stabilizing $\mathrm{Ro}_{Q}$ increases, as though turbulence in the free shear layer were being damped. The range of Rotation numbers was too limited at the higher Reynolds numbers to draw any strong conclusions.

Nilsen and Anderson (1990) attempted to compute the higher Reynolds number flows for a step diffuser of infinite aspect ratio and $\mathrm{AR}=2$, like one used in our experiments. They used the RANS equations with an algebraic, second order Reynolds stress closure model and obtained fair agreement with the experimental results as shown in Fig. 14, at the higher Reynolds numbers.

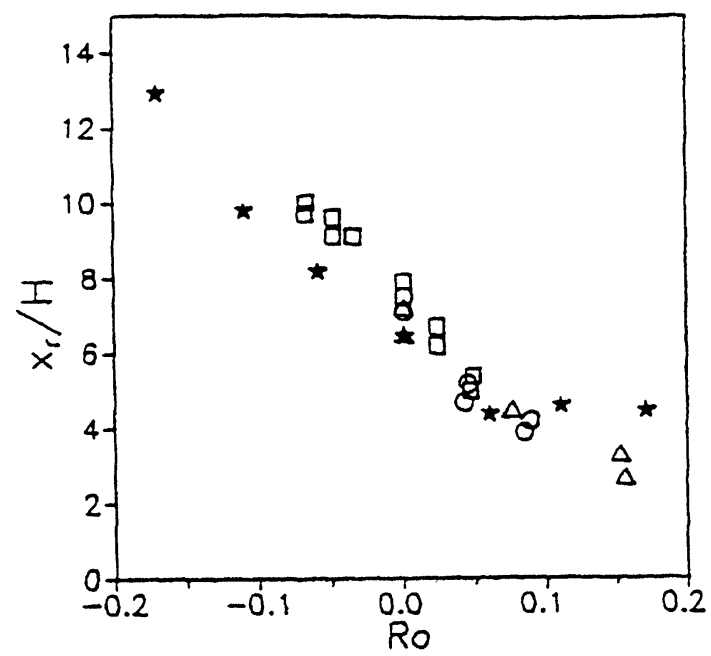

FIGURE 14 Reattachment distance, $X_{\mathrm{r}} / H \quad(H=b$, step height) versus Rotation number from Nilsen and Anderson (1990). Data (open symbols) of Rothe and Johnston (1979), $\mathrm{AS}=15$, high $\mathrm{Re}$ numbers. $\star$, Computed using algebraic second-moment Reynolds stress model, $\mathrm{AS}=\infty$.
However, their results indicated a leveling (saturation) effect at high values of destabilization Rotation number. This effect was not seen in the Rothe and Johnston data, but a similar effect was seen in the wall friction data for the pressure (destabilized) side of the rotating channels (upper branch in Fig. 8). In both cases, this saturation of the destabilizing effect of rotation was attributed to a rather complex interation of the quasi stationary roll cell structures with the destabilizing influence of rotation on the production terms in the equations for the evolution of the turbulence Reynolds stresses (derived from the Navier-Stokes equations). A detailed examination of their papers is suggested for modelers interested in developing practical methods of computation.

\section{CONCLUDING REMARKS}

An attempt was made to conduct a coherent discussion of two of the effects of system rotation (secondary flow and stabilization) on flows in geometrically simple cases, rectangular channels and diffusers. The effects rotation in regard to secondary flows in end-wall boundary layers were already fairly well understood 25-30 years ago. However, new, basic data on the stabilizing/ destabilizing effects of Coriolis force on boundary and free shear layers has been obtained since 1970, and thus primary emphasis was placed on discussion of the latter effects in this paper.

Although the review has concentrated on experiments in simple basic flows, the discussion indicates where and how these results are related to practical flows in pump and compressor impellers and turbine rotors. Predictions of complex flows in rotors is a major objective of engineers and designers, and some have attempted to empirically model the stabilizing/destabilizing effects for inclusion in codes that numerically solve the Reynolds averaged Navier-Stokes equations. The state of the art in practical turbulence modeling for turbomachines, or for that matter even simple rotating flows such as straight channels and diffusers with 
wall boundary layers is still rather primitive, and thus this topic is better left to a substantial review at a later time, hopefully sooner than 25 years from now.

\section{References}

Andersson, H.I. and R. Kristoffersen (1993) Reynolds-stress budgets in rotating channel flow, 9 th Symposium on Turbulent Shear Flows, Kyoto, Japan, August 16-18, 1993, pp. 29.4.129.4 .6$.

Bidokhti, A.A. and D.J. Tritton (1992) The structure of a turbulent free shear layer in a rotating fluid, Journal of Fluid Mechanics, 241, 469-502.

Bradshaw, P. (1969) The analogy between streamline curvature and buoyancy in turbulent shear flow, Journal of Fluid Mechanics, 36 (part 1), 177-191.

Dean, R.C. Jr. (1968) On the unresolved fluid dynamics of the centrifugal compressor, Paper delivered at ASME Gas Turbine Division Conference, Washington, D.C., March 17 $21,1968$.

Fowler, H.S. (1968) The Distribution and stability of flow in a rotating channel, Journal for Engineering for Power, Trans. ASME, 90, 229-236.

Hart, J.E. (1971) Instability and secondary motion in a rotating channel flow, Journal of Fluid Mechanics, 45, 341-352.

Hill, P.G. and I.M. Moon (1962) The effects of coriolis on the turbulent boundary layer in rotating fluid machines, Report 69, Gas Turbine Laboratory, M.I.T., June 1962.

Ibal, G. and P.N. Joubert (1992) A study of combined effects of rotation and adverse pressure gradient on turbulent boundary layer flows: Part 1 - Mean flow results, Proceedings of the 11th Australasian Fluid Mechanics Conference, U. of Tasmania, Hobart, Australia, 14-18 December 1992, pp. $825-828$.

Ito, H. and K. Nambu (1971) Flow in rotating straight pipes of circular cross section, Journal of Basic Engineering, Trans. ASME, Series D, 93, 383-394.

Johnston, J.P. and S.A. Eide (1976) Turbulent boundary layers on centrifugal compressor blades: Prediction of the effects of surface curvature and rotation, Journal of Fluids Engineering, Trans. ASME, 98, 374-387.

Johnston, J.P. (1974) The effects of rotation on boundary layers in turbomachine rotors, Fluid Mechanics, Acoustics, and Design of Turbomachinery, Eds. B. Lakshminarayna, W. R. Britsch and W. S. Gearhart, pp. 207-249, NASA SP-304.

Johnston, J.P., R.M. Halleen, and D.K. Lezius (1972) Effects of spanwise rotation on the structure of two-dimensional fully developed turbulent channel flow, Journal of Fluid Mechanics, 56, 533-557

Kikuyama, K.M., M. Murakami, and H. Matsumoto (1980) Flows in rotating diffusers (effects of diffuser angle), Bulletin of the Japan Society of Mechanical Engineering, 23(179), 665671.

Kikuyama, K., M. Murakami, S. Oda, and K. Gomi (1987) Pressure recovery of rotating diffuser with distorted inflows, Journal of Fluids Engineering, Trans. ASME, 109, 114-120.

Koyama, H.S., E. Tamura, and T. Saito (1989) Effects of coriolis force on developing turbulent channel flow, 11th Australasian Fluid Mechanics Conference, U. of Tasmania Hobart, Australia, 14-18 December 1992, pp. 12.17-12.20.
Koyama, H.S., S. Masuda, I. Agriga, and I. Watanabe (1979) Stabilizing and destabilizing effects of Coriolis force on twodimensional fully developed turbulent channel flow, Journal of Engineering for Power, Trans. ASME, 101, 23-31.

Koyama, H.S., K. Kitagawa, and K. Chen (1995) Secondary flow and stability effects of Coriolis force on a turbulent boundary layer in a rotating channel, 10th Symposium on Turbulent Shear Flows, Pennsylvania State U., University Park, PA, August 14-16, 1995, pp. 8.7-8.12.

Kristoffersen, R. and H.I. Andersson (1993) Direct simulations of low Reynolds number turbulent flow in a rotating channel, Journal of Fluid Mechanics, 256, 163-197.

Lezius, D.K. and J.P. Johnston (1976) Roll-cell instabilities in rotating laminar and turbulent channel flows, Journal of Fluid Mechanics, 77, 153-175.

Matsubara, M. and S. Masuda (1991a) Turbulent spots in a rotating turbulent boundary layer, Advances in Turbulence 3 , Eds. Johansson and Alfredson, pp. 204-210, SpringerVerlag, Berlin, Heidelberg, 1991.

Matsubara, M. and S. Masuda (1991b) Three-dimensional instability in rotating boundary layer, ASME, FED-Vol. 114, Boundary Layer Stability and Transition to Turbulence, pp. 103-107.

Moon, I.M. (1964) Effects of Coriolis force on the turbulent boundary layer in rotating fluid machines, Report 74, Gas Turbine Laboratory, M.I.T., June 1964.

Moore, J. (1967) Effects of Coriolis on the turbulent flow in rotating channels, Report 89, Gas Turbine Laboratory, M.I.T., January 1967.

Moore, J. (1973) A wake and an eddy in a rotating radial passage, Part 1: Experimental observations, Journal of Engineering for Power, Trans. ASME, 95, 205-212.

Miyake, Y. and T. Kajishima (1986) Numerical simulation of the effects of Corolis force on the structure of turbulence, Bulletin of the Japan Society of Mechanical Engineering, 29, 3341-3351.

Murakami, M. and K. Kikuyama (1976) Effects of rotation on diffuser flow, Bulletin of the Japan Society of Mechanical Engineering, 19(137), 1294-1301.

Nilsen, P.J. and H.I. Andersson (1990) Rotational effects on sudden-expansion flows, Engr. Turbulence Modeling and Experiments, Eds. Rodi and Ganic, pp. 65-72, Elsevier Science Pub. Co.

Rothe, P.H. and J.P. Johnston (1975) Stall in rotating twodimensional diffusers, Journal of Fluids Engineering, Trans. ASME, 97, 252-253.

Rothe, P.H and J.P. Johnston (1976) Effects of system rotation on the performance of two-dimensional diffusers, Journal of Fluids Engineering, Trans. ASME, 98, 422-430.

Rothe, P.H. and J.P. Johnston (1979) Free shear layer behavior in rotating systems, Jouurnal of Fluids Engineering, Trans. ASME, 101, 117-120.

Wagner, R.E. and Velkoff, (1972) Measurements of secondary flows in a rotating duct, Journal of Engineering for Power, Trans. ASME, 94, 261-270.

Watmuff, J.H., H.T. Witt, and P.N. Joubert (1985) Developing turbulent boundary layers with system rotation, Journal of Fluid Mechanics, 175, 405-448.

Witt, H.T. and P.N. Joubert (1985) Effect of rotation on a turbulent wake, 5th Symposium on Turbulent Shear Flows, Ithica, NY, August 7-9, pp. 21.25-21.30 


\section{ait \\ ENERGY MATERIALS}

M A N E Y publishing

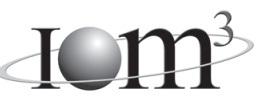

\section{Materials Science \& Engineering for Energy Systems}

Maney Publishing on behalf of the Institute of Materials, Minerals and Mining

The Institute of Materials, Minerals \& Mining

Economic and environmental factors are creating ever greater pressures for the efficient generation, transmission and use of energy. Materials developments are crucial to progress in all these areas: to innovation in design; to extending lifetime and maintenance intervals; and to successful operation in more demanding environments. Drawing together the broad community with interests in these areas, Energy Materials addresses materials needs in future energy generation, transmission, utilisation, conservation and storage. The journal covers thermal generation and gas turbines; renewable power (wind, wave, tidal, hydro, solar and geothermal); fuel cells (low and high temperature); materials issues relevant to biomass and biotechnology; nuclear power generation (fission and fusion); hydrogen generation and storage in the context of the 'hydrogen economy'; and the transmission and storage of the energy produced.

As well as publishing high-quality peer-reviewed research, Energy Materials promotes discussion of issues common to all sectors, through commissioned reviews and commentaries. The journal includes coverage of energy economics and policy, and broader social issues, since the political and legislative context influence research and investment decisions.

\section{CALL FOR PAPERS}

Contributions to the journal should be submitted online at http://ema.edmgr.com

To view the Notes for Contributors please visit: www.maney.co.uk/journals/notes/ema

Upon publication in 2006, this journal will be available via the Ingenta Connect journals service. To view free sample content online visit: www.ingentaconnect.com/content/maney

For further information please contact:

Maney Publishing UK

Tel: +44 (0)113 2497481 Fax: +44 (0)1132486983 Email: subscriptions@maney.co.uk

or

Maney Publishing North America

Tel (toll free): 8662975154 Fax: 6173546875 Email: maney@maneyusa.com

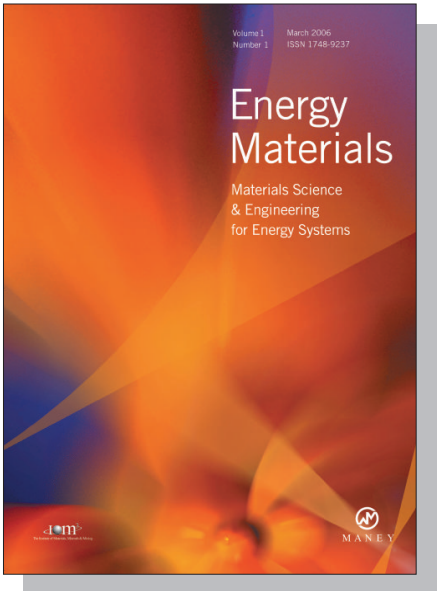

EDITORS

Dr Fujio Abe

NIMS, Japan

Dr John Hald, IPL-MPT, Technical University of Denmark, Denmark

Dr R Viswanathan, EPRI, USA

\section{SUBSCRIPTION INFORMATION}

Volume 1 (2006), 4 issues per year

Print ISSN: 1748-9237 Online ISSN: 1748-9245

Individual rate: $£ 76.00 / U S \$ 141.00$

Institutional rate: $£ 235.00 /$ US $\$ 435.00$

Online-only institutional rate: $£ 199.00 / U S \$ 367.00$

For special $\mathrm{IOM}^{3}$ member rates please email

subscriptions@maney.co.uk

\section{For further information or to subscribe online please visit www.maney.co.uk}



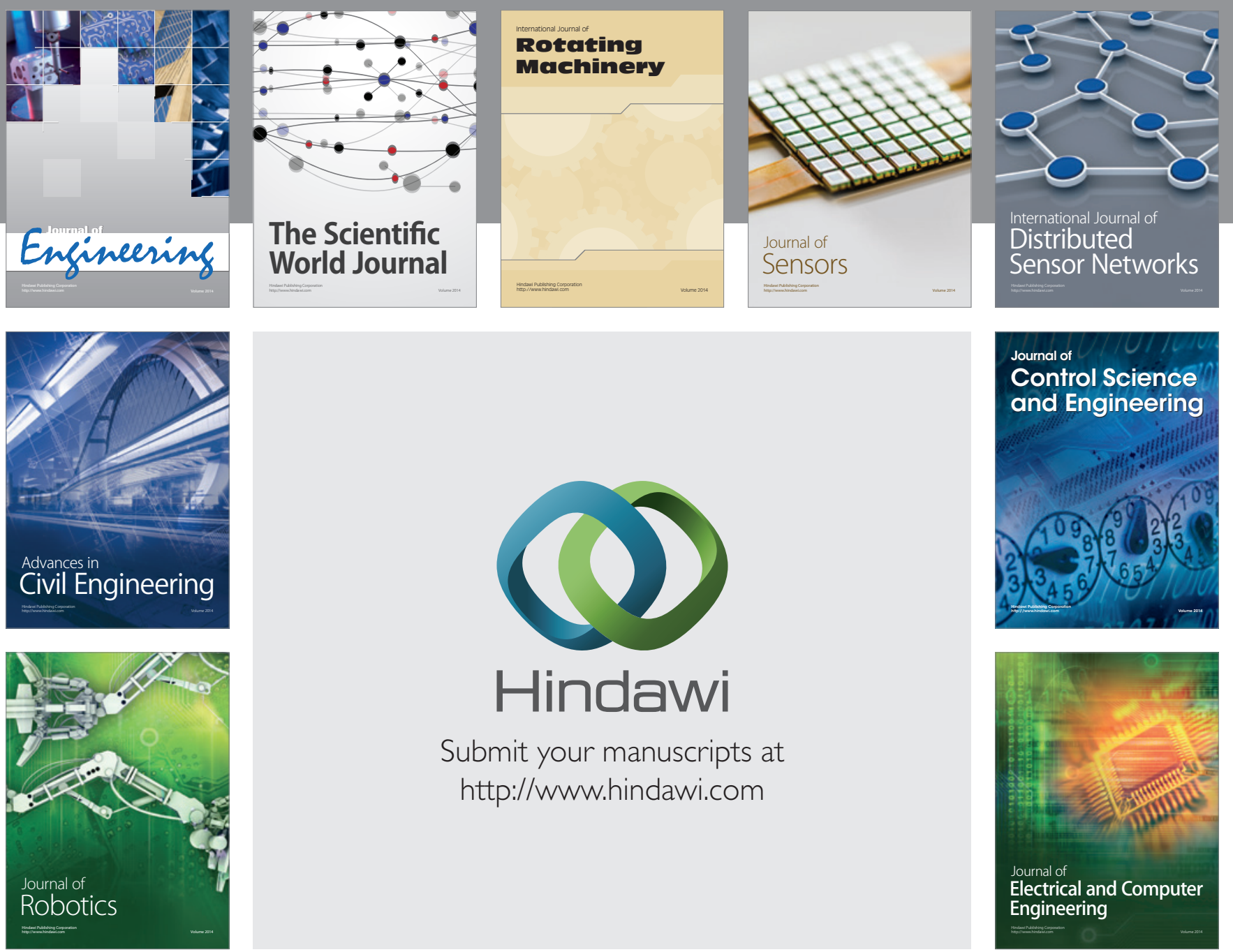

Submit your manuscripts at

http://www.hindawi.com
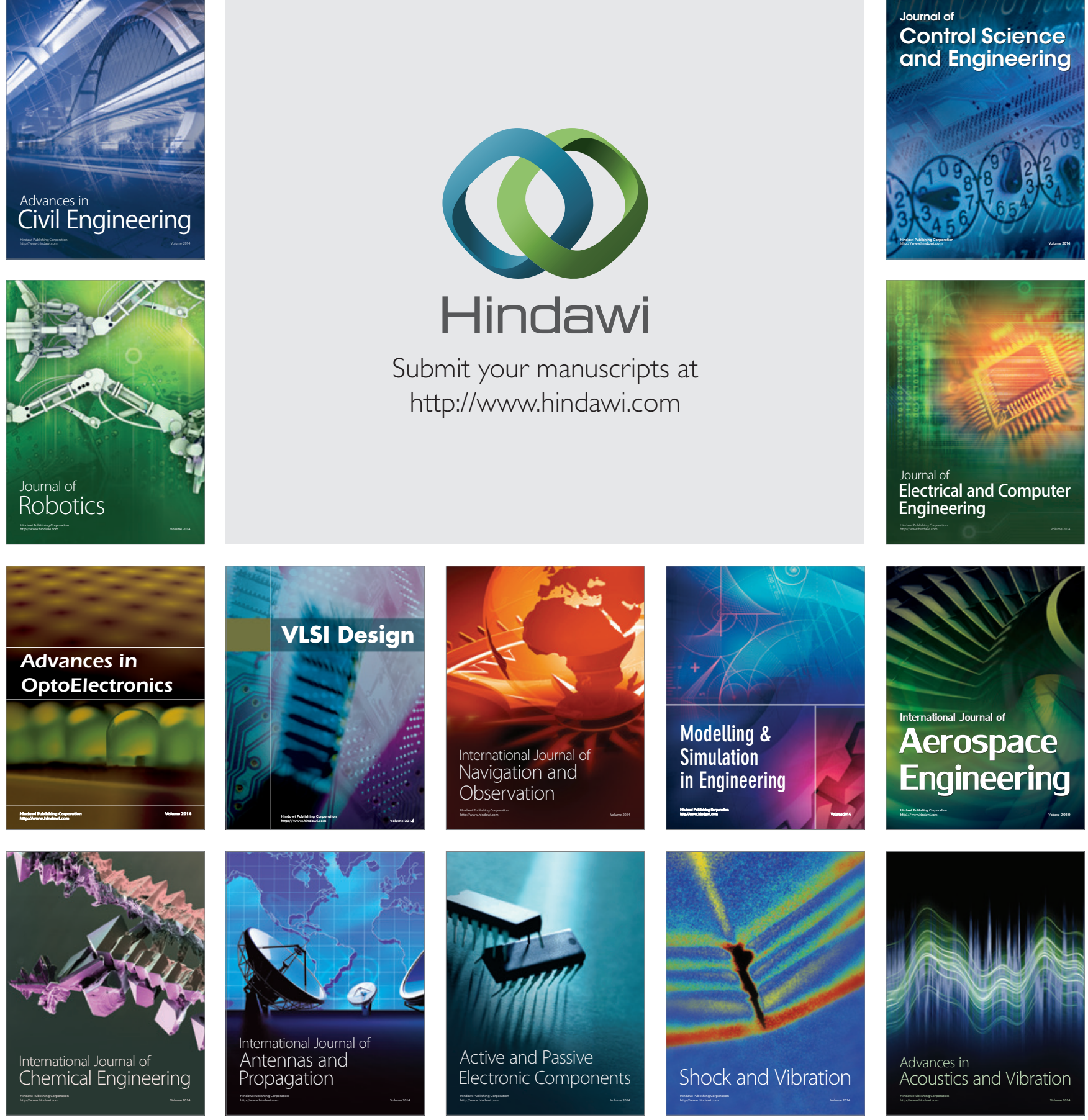